\title{
The following abstracts were presented as posters at the 2012 NEI Global Psychopharmacology Congress
}

\section{DISCLAIMER}

The material presented in this section contains the abstracts submitted as part of the poster session at the 2012 NEI Global Psychopharmacology Congress, San Diego, CA, USA, 18-21 October 2012.

The abstracts have not been peer reviewed as part of the standard processes of CNS Spectrums and may contain promotional content that does not represent the views or policies of the journal or publisher. The abstracts are not approved for continuing education credit. Poster content and the views expressed therein are those of the presenting entity and not the Editors of CNS Spectrums, Cambridge University Press or of the Neuroscience Education Institute. Authors supply a standard disclosure form to the conference organizers which they retain. 


\title{
Abstracts
}

The following abstracts were presented as posters at the 2012 NEI Global Psychopharmacology Congress.

Congratulations to the Scientific Poster Competition winners of the 2012 NEI Global Psychopharmacology Congress. 1st Place: Sirid Kellermann, PhD, MBA (PS-113); 2nd Place: John A Bilello, PhD (PS-124); 3rd Place: Jonathan Fellus, MD (PS-109).

\section{PS-101}

Clinical and Socio Demographic Profile of Epilepsy Cases in Various Tertiary Care Hospitals in Mangalore City of South India

Nitin Joseph, MBBS, MD, PGDFM

\begin{abstract}
:
Introduction: Epilepsy is a chronic neurological disorder that affects people of all ages. Around 50 million people worldwide have epilepsy. Nearly $85 \%$ of the people with epilepsy are found in developing regions. It is estimated that India alone has approximately 8-10 million people suffering from epilepsy. Although epilepsy has been recognized since ancient times, a scientific understanding of the problem is recent. Reason for lack of research in epilepsy probably could be because unlike many other disorders, instances of death directly due to epilepsy are few. The exact difference in the number of cases and the causes of seizures between urban and rural areas is also not clearly known. Studies have revealed that in most SEAR countries, nearly half of people with epilepsy either do not receive systematic treatment and among those who are on treatment, nearly $40 \%-70 \%$ drop out at various stages of treatment, resulting in the recurrence of seizures. With this background this study was done to find out the socio demographic profile of epilepsy cases, etiological factors of epilepsy, management of epilepsy and to find out the compliance rate with treatment.
\end{abstract}

Materials and methods: This hospital based retrospective study was done in four tertiary care hospitals in Mangalore city of south India during May 2012. Clinical records of 196 cases admitted with epilepsy in the past 5 years were reviewed.

Results: Most patients were aged between 5 to 15 years $40(20.4 \%)$. Mean age of onset of epilepsy was $19.9 \pm 18.8$ years. Majority of cases were males $119(60.7 \%)$. Majority of patients $(86.7 \%)$ were unskilled workers and majority $133(67.9 \%$ ) cases were from urban areas. $82.9 \%$ patients earned less than 20 per month. Family history of epilepsy was present in $8.4 \%$ cases. Commonest type of epilepsy was the generalized tonic clonic variety seen in $153(78.1 \%)$ cases. Clinical features like uprolling of eyes, loss of consciousness, salivation, urinary bladder incontinence, drowsiness and muscle flaccidity were seen significantly more among GTCS cases $(P=0.000)$. Cause of epilepsy was idiopathic in majority $130(66.3 \%)$ of cases. Commonest cause of secondary epilepsy was head trauma $16(24.2 \%)$ followed by CNS infections $11(16.7 \%)$. Phenytoin was most commonly anti epileptic drug $141(71.9 \%)$. Compliance rate with treatment was found to be $81.9 \%$.

Conclusion: Socio demographic profile showed more cases among children, males, unskilled workers, low socio economic groups and from urban areas. Commonest cause of secondary epilepsy was trauma followed by infection of central nervous system. GTCS was the commonest type of epilepsy. Phenytoin was the drug most commonly used AED. Treatment with AED was found to be irregular in 18.1\% cases. Understanding of risk factors will help in improvement of case management. Educating the patients is will help in further minimizing the non-compliance rate with treatment.

PS-102

Spectroscopically Visualized Reaction Intermediates in P450 Enzyme Reactions James Arthur Halgrimson

\begin{abstract}
Long noted since their discovery in the 1960's, P450 enzymes are believed to play a key role in the oxidation of hydrocarbons. Most pharmaceuticals are thought to be metabolized in the human body by P450 enzymes via high energy reactions within the P450 active site. A key reaction is the hydroxylation of hydrocarbons, which is thought to result in increased substrate hydrophilicity, thus perpetuating the excretion of metabolic products. These reactions are thought to be due to the interaction of an iron-oxo radical cation species, compound I, and hydrocarbon substrates within the P450 active site. Compound I is a well noted high energy intermediate within the P450 reaction cycle that is thought to possess energy capable of abstracting hydrogen and thus hydroxylating hydrocarbons. This study demonstrates evidence of P450's formation of compounds I and II utilizing X-ray absorption fluorescent spectroscopy (EXAFS) and UV visable spectroscopy under controlled mixing conditions with peroxinitrite in a P450 enzyme isolated from the thermophilic archaeabacterium Sulfolobus solfactaricus. The results demonstrate evidence of the first visualized Compound I and Compound II species within a P450 enzyme.
\end{abstract}


PS-103

Multidimensional Complexity in the Dementias: Diagnosis, Stage, Comorbidity, and Neuropsychiatric Symptoms Tatiana Sadak, PhD, PMHNP; Jodie Katon, PhD; Soo Borson, MD

\begin{abstract}
:
Objective: To further the understanding of multimorbidity in patients with dementia, using clinical subtype, stage, neuropsychiatric symptoms, and selected comorbidities to model associations and interactions.
\end{abstract}

Background: Assessment and management of the health care needs of persons with dementia are complicated by heterogeneity in diagnosis, disease severity, neuropsychiatric symptoms (NPS) and medical comorbidities.

Design/Methods: Cross-sectional analysis of Uniform Data Set variables from the National Alzheimer's Coordinating Center repository. Subjects were 3,976 patients, aged 65+, with dementia of a single etiology (Alzheimer's [AD], Lewy body [DLB], behavioral-variant frontotemporal [bvFTD], vascular [VaD]) or mixed etiologies (AD/DLB, AD/VaD). NPS most strongly associated with caregiver burden (agitation/aggression, depression/dysphoria, anxiety, irritability/ lability) were scored using the Neuropsychiatric Inventory, and major comorbid conditions (cardiovascular disease, cerebrovascular disease, diabetes) were identified using a structured checklist. Descriptive statistics and logistic regression were used to identify the prevalence and relative odds of NPS by dementia type, stage and the presence of target comorbidities. The interaction effects of dementia stage and comorbid conditions on NPS were tested using Likelihood Ratio Tests, using AD as the reference group for all comparative analyses.

Results: The average overall prevalence of NPS was approximately $40 \%$, and was stage-dependent for all diagnoses. DLB, either alone or in combination with AD, was associated with significantly increased odds of depression/dysphoria and anxiety [OR 1.63 (95\%CI 1.27-2.09) to 1.76 (95\%CI 1.11-2.78)]; bvFTD was associated with increased agitation/aggression [OR 1.48 (95\%CI 1.09-2.01) and anxiety 1.40 (95\%CI 1.04-1.90)]. The association of dementia diagnosis with NPS appeared to be modified by the presence of cardiovascular disease, cerebrovascular disease and diabetes.

Conclusions: This study confirms previously reported associations of specific NPS with different dementia subtypes, but adds two novel elements: DLB dominates NPS profiles when present even as a secondary diagnosis, and comorbid medical illness may alter NPS presentations in neurodegenerative dementing diseases.

Data from: National Alzheimer's Coordinating Center (NACC) database [U01 AG016976]. Funded by: John A. Hartford Foundation's Building Academic Geriatric Nursing Capacity Award Program.

PS-104

Incidence of Tardive Dyskinesia in an Outpatient Population During Long-Term Teatment with Second Generation Antipsychotics

Jefferson K. Davis, MD, MA

Study Objective: to determine the incidence of tardive dyskinesia due to long-term exposure to SGAs in an outpatient population taking concomitant psychotropic medications.

Method: All patients who were seen in an outpatient practice over a three-month period and were currently taking SGAs for a minimum duration of 6 months were observed for dyskinetic signs and administered the AIMS. A total of 105 patients met the criteria.

Results: The average duration of exposure to SGAs was 5.7 years. Primary diagnoses included major depressive disorder without psychosis (58), major depressive disorder with psychotic features (4), bipolar disorder without psychosis (25), bipolar disorder with psychotic features (9), schizoaffective disorder (6), schizophrenia (1) and pervasive developmental delay (2). 102 patients were taking concomitant mood stabilizers and/or antidepressants and 3 were taking only concomitant benzodiazepines. Six patients had prior or current exposure to first generation antipsychotics. Five patients had a positive AIMS score $(>1)$ of which three patients manifested overt symptoms of orobuccal TD for an incidence of $4.8 \%$ and $2.9 \%$ respectively. Two patients with overt TD experienced profound symptomatic relapse when the SGA was discontinued and therefore elected to continue the SGA.

Conclusions: The incidence of TD in this population was low, which is consistent with most but not all studies of TD in patients treated with SGAs. What makes this study unique is the duration of exposure to SGAs in a population that consists primarily of mood disorders and is exposed to concurrent psychotropic medication.

Educational Objectives: After studying this material, the participant should be better able to describe the incidence of tardive dyskinesia in an outpatient psychiatric population that consists primarily of mood disorder diagnoses and is treated with concomitant medication. This study helps to fill in a gap in the knowledge base for the large number 
of psychiatrics who practice in the private setting or in clinics where they treat people with chronic disorders, in which SGAs are prescribed in conjunction with other psychotropics.

Funding: no funding

\section{PS-105}

Kava Intoxication: Acute Movement Disorders in Patient with Kava Addiction Liudmila Lobach, MD; Bryan Chambliss, MD

Abstract: Kava (Piper Methysticum) is a psychoactive herbal drink widely used by people of the South Pacific nations to relax and as an ice-breaker in marital and other social ceremonies. In the United States, it is used primarily for sleep and anxiolysis, reportedly without addiction.

This patient used large amounts of Kava daily and developed classic dermatologic, hematologic, central nervous system and gastrointestinal side effects. On several occasions, he developed acute dystonia and choreoathetosis after using 1-2 months' supply of Kava in one day. Patient also was treated with conventional medications.

The present report describes the effects of acute Kava intoxication from heavy chronic consumption and discusses the pharmacological and clinical manifestation of Kava toxicity.

Since Kava use is gaining popularity (US sale is $\$ 17$ million/year), the need for awareness of effects are highlighted.

Case Description:

- A 55 year-old Caucasian male with a history of bipolar disorder, type II and alcohol dependence presented for treatment-resistant depression.

- At the time of the observed symptoms patient was taking venlafaxine XR $275 \mathrm{mg}$ daily, gabapentin $300 \mathrm{mg}$ twice a day, risperidone $1.5 \mathrm{mg}$ at bed time, but had not revealed excessive Kava use.

- Patient complained of lethargy, poor appetite, nausea and loss of 20 pounds, dry skin (thick, scaly, gray calluses covering palms), grayish "corpse-like appearance" of face, bruises on face and arms from accidents at work. He attributed the above to side effects and loss of efficacy of medications.

- Four months before Kava use was revealed, laboratory results showed a low white blood cell, lymphocyte count and platelet volume. Patient was referred to hematology.

- Patient admitted to daily kava use and denied alcohol use after confrontation about increased frequency of accidents at work.

- He said that Kava relaxed and made him less "frightened of clients". Patient consumed 1 to 2 week supply daily and 1 to 2 month's supply at once.

- Drinking Kava without diluting it caused immediate vomiting. Excessive consumption, "made him drunk," accompanied by staggering around and falling to the ground. While intoxicated with Kava, patient would make paranoid accusations and talk in the voice of a little boy. Two episodes of intoxication were accompanied by "odd movements" both certain dystonic and likely choreoathetotic movements . Kava was tapered and patient stopped using it but relapsed two weeks after. He felt that he was addicted to Kava even though Kava was reported to be a non-addictive drug. Patient later successfully completed Kava detoxification with clonazepam taper. Gray scaly calluses on palms, discoloration of face, ataxia, and all laboratory abnormalities slowly returned to normal with no further episodes of movement disorders.

Symptoms presented in this case, including dystonia and choreoathetosis, could be attributed to two major Kava mechanisms of action:

1. Pharmacodynamic: (direct effect of Kava on receptors). Effect on dopamine level is dose-dependent: Kava in recommended doses decreases dopamine levels and in significantly large doses increases dopamine levels leading in this case to paranoia, dystonia and choreoathetosis.

Potentiation of CABA-A: similarity to benzodiazepines could contribute to the potential addictive effect of Kava. One should avoid using Kava with alcohol and benzodiazepines to minimize augmentation of sedative effects.

2. Pharmacokinetic: As indicated in a recent study, Kava has a high potential for causing drug interactions through inhibition of CYP450 enzymes which suggests potentiation of EPS in the present report because Kava significantly inhibits CYP 2D6 and 3A4 which are involved in risperidone metabolism. 
Adverse reactions, apparently due to a conventional medicine, might be due to a herbal medicine or to the interaction between herbal medicines and conventional drug, particularly when the health professional is unaware of the extent of a patient's self-medication with an alternative therapies.

Physicians should be aware of Kava's common and toxic side effects, drug interactions and laboratory manifestations of Kava abuse to be able establish correct diagnosis and provide effective treatment.

\title{
PS-106
}

Antipsychotic use and Cardiometabolic Health: A Quality Improvement Project Led by Psychiatry Residents Ksenia Nawrocki, MD; Jayesh Kamath, MD, PhD

\begin{abstract}
:
Study Objectives: Use of antipsychotics (AP) has been on the rise secondary to broader FDA approved indications which now includes bipolar disorder and adjunctive treatment for depression/anxiety. Use of APs, however, has also been associated with higher risk of serious medical consequences. A recent study suggested that antipsychotic use is independently associated with cardiometabolic risks, even after adjusting for patient's lifestyles characteristics. Several genetic, psychosocial and illness related factors might play a role in increasing cardiometabolic risks. Such factors might include socioeconomic status, comorbidities, and non-compliance with treatment/monitoring recommendations.
\end{abstract}

In the present psychiatry residents-led project, we investigated AP use, their indications, profiles of patients receiving AAPs and monitoring of cardiometabolic health conducted by outpatient clinicians. This project was conducted by third year (PG3) psychiatry residents in the outpatient psychiatry clinic at the University of Connecticut Health Center (UCHC).

Methods: An assessment form was generated for the project and was approved by the UCHC Institutional Review Board (IRB). The assessment form included following information: patient demographic factors (age, gender, education, race and ethnicity), APs and indications for their use, comorbidities including cardiovascular issues and diabetes, familial risk factors, current monitoring of cardiometabolic health (as recommended by the American Psychiatric Association/American Diabetes Association consensus statement, 2004).

Patients receiving APs were randomly selected from the case load of six PGY3 psychiatry residents at the beginning of their third year in the outpatient clinic. Using the assessment form mentioned earlier, a retrospective chart review was conducted by the residents on their selected cases.

Results: A total of 93 patient charts were reviewed (46 males and 47 females). Patients were primarily white $(n=61)$. More than half $(55 \%)$ had high school education or less. Risperidone $(n=30)$ was the most frequently prescribed antipsychotic, followed by quetiapine $(n=23)$ and aripiprazole $(n=20)$. The indications included psychosis $(n=29)$, bipolar disorder $(n=28)$, adjunctive treatment for depression/ anxiety $(n=15)$ and off-label indications $(n=21)$ such as impulse control disorder. A total of 12 patients were receiving two APs simultaneously. Approximately two third of patients carried a secondary psychiatric diagnosis and almost all (93\%) were receiving concomitant psychotropic medications. Approximately one third of patients $(n=29)$ had known cardiovascular comorbidities and similar number of patients $(n=24)$ were diagnosed with diabetes. Preliminary analyses showed significant gaps in monitoring of cardiometabolic health of these patients with lack of coordination between the psychiatric prescriber and primary care clinicians.

Discussion \& Conclusions: APs are being prescribed more often for non-psychotic indications with significant gaps in monitoring of cardiometabolic health. A second-phase of the project is underway to rigorously assess riskbenefit ratio when prescribing APs and to improve monitoring as recommended by the APA/ADA guidelines. This unique psychiatry resident-driven project suggests that residents can successfully lead such projects to evaluate self-prescription patterns and to improve well-being of their patients.

Educational objectives: After studying the project findings, the participants should be able to describe the characteristics of patients receiving antipsychotics and the indications for their use in a typical academic outpatient psychiatry clinic. The participants should also be able to delineate comorbidities and preexisting cardiometabolic risk factors in patients receiving antipsychotics and ways to appropriately coordinate monitoring of medical consequences associated with antipsychotic use.

Funding: no funding 
PS-107

Verapamil for the Treatment of Clozapine-Induced Persistent Sinus Tachycardia

Abhishek Rai, MD; Piyush Das, MD; Preetha Sharone Kuppuswamy, MBBS; J. Michael Bostwick, MD

Abstract:
STUDY OBJECTIVE:
- Is tachycardia associated with use of clozapine, dose related?
- Can Verapamil be used to treat clozapine-induced tachycardia?

CASE REPORT: A 17-year-old South Asian male with a medical history significant for asthma and a strong family history of schizophrenia was brought to our emergency room (ER) by police for bizarre behaviors including refusal to wear clothes and perseveration on his sexual orientation. Sertraline had been started a few weeks prior to this ER visit for anxiety. At an outside hospital to which he had been transferred due to our psychiatric unit being full, he was started on aripiprazole $5 \mathrm{mg}$ daily in addition to the sertraline whose dose was increased to $100 \mathrm{mg}$ daily. After dismissal, he continued to exhibit flight of ideas, pressured speech, grandiose delusions and mood lability, as well as obsessional ideas of a sexual nature, disorganized thought process, mood-incongruent affect, and paranoia directed at his mother. After re-hospitalization, sertraline and aripiprazole were discontinued, and risperidone was started and titrated to $6 \mathrm{mg}$ at bedtime. Divalproex sodium was also added for prominent mood instability and titrated up to $250 \mathrm{mg}$ twice daily. Other organic causes for his psychosis were ruled out, although routine laboratory studies did reveal an elevated TSH of 26.1 with normal free T3, T4 and TPO antibody, levothyroxine $125 \mathrm{mcg}$ daily was initiated. After a month in the hospital, his mental status had improved to the point that he could be discharged.

The improvement didn't last. Only a month later he was rehospitalized for safety concerns, avolition, increasing sexual thoughts, extreme castration anxiety, and poor self-hygiene. Risperidone was discontinued and aripiprazole tried again, this time titrated to a dose of $25 \mathrm{mg}$ daily without benefit. Aripiprazole was replaced by quetiapine $600 \mathrm{mg}$, again without much improvement. Quetiapine was discontinued, and clozapine gradually titrated to a dose of $300 \mathrm{mg}$ daily. At dismissal, his psychotic symptoms had markedly decreased and he was able to attend to his hygiene and dress. In the outpatient arena, he was noted to have persistent sinus tachycardia on pulse checks during clinic visits (also recorded at home) without subjective symptoms. When a cardiology workup that included ECG, Holter monitor and echocardiogram ruled out any other explanation for the tachycardia than clozapine, he was cross-titrated to ziprasidone, reaching a dose of $120 \mathrm{mg}$ daily without response. He was switched to asenapine, $5 \mathrm{mg}$ twice daily, which also proved ineffective.

With one antipsychotic trial after another having proven ineffective and the patient's psychotic symptoms continuing to worsen, he was hospitalized a fourth time. After loxapine, $35 \mathrm{mg}$ daily, which replaced asenapine, failed to help much, the treatment team decided to try clozapine again, given that it was the only antipsychotic to which his psychosis had satisfactorily responded. The team simultaneously decided to manage the tachycardia pharmacologically. Given his asthma history, the decision was made to avoid beta-blockers. A literature review revealed that the calcium channel blocker verapamil, effective for tachycardia, had also been shown to have efficacy for some symptoms of schizophrenia and bipolar disorder. On immediate release verapamil, $40 \mathrm{mg}$ three time's daily, the patient experienced no untoward effects, and his heart rate entered the normal range. The shortacting verapamil formulation was switched to an extended release formulation at $180 \mathrm{mg}$ once daily to maximize control of the tachycardia and ease compliance. On the combination of clozapine and extended-release verapamil, the patient's psychotic symptoms significantly improved and he was dismissed on clozapine $150 \mathrm{mg}$ at bedtime. In subsequent months the outpatient clozapine dose was increased to $400 \mathrm{mg}$ as an outpatient and he has not required further hospitalization.

RESULTS: On reviewing the vital sign charts, tachycardia has stayed in remission with the use of verapamil.

CONCLUSIONS: In patients on clozapine therapy, tachycardia can occur with or without myocarditis. In actuality, clozapine increases the heart rate in majority of patients who take it and about one quarter of those on therapeutic doses manifest an average increase of 10-15 beats/minute. Beta-adrenergic agonists are effective tools for managing clozapine-induced tachycardia. As an alternative to a beta blocker in our asthmatic patient, we elected to try verapamil in hopes of taking advantage not only of its potential for suppressing tachycardia but also of its documented efficacy as an augmenting agent against psychotic symptoms in patients with schizophrenia or bipolar disorder.

\section{EDUCATIONAL OBJECTIVE:}

- Clozapine induced sinus tachycardia is dose related.

- Verapamil can be used effectively to treat clozapine induced sinus tachycardia (especially in asthmatic patients, in whom use of beta blockers is restricted) -Verapamil has relevance in augmenting clozapine therapy for schizophrenia, especially when prominent negative symptoms and affective disturbances are present. 


\title{
PS-108
}

PRISM Registry: A Novel Tool to Estimate the Prevalence of Pseudobulbar Affect Symptoms

Jonathan Fellus, MD; Daniel Kantor, MD; Randall E Kaye, MD; Eileen Hernandez

\author{
Abstract: \\ Study objectives: To estimate the prevalence of pseudobulbar affect (PBA) for six commonly associated \\ neurological conditions in a clinical setting utilizing a registry.
}

Method: PBA is a neurological condition that occurs secondary to a variety of otherwise unrelated neurological diseases or injuries and manifests as involuntary, frequent, and disruptive outbursts of crying and/or laughing Although prevalence estimates suggest PBA impacts between 1.5 and 2 million people in the US, it is generally thought to be under-recognized and undertreated because patients, caregivers, and clinicians are unfamiliar with the disorder.

Disease registries are powerful tools for obtaining epidemiologic data. The PBA Registry Series (PRISM) was initiated in 2011, and designed to capture data in patients with conditions predisposing to PBA. Participating centers join via a centralized web portal and register with a central Institutional Review Board (IRB). Investigators are asked to enroll at least 20 patients with Alzheimer's disease (AD), amyotrophic lateral sclerosis (ALS), multiple sclerosis (MS), Parkinson's disease (PD), stroke, or traumatic brain injury (TBI). Consenting patients (or their caregivers) complete the Center for Neurologic Study-Lability Scale (CNS-LS), and a QOL measure. Demographic data, date of primary-disease diagnosis, and use of concomitant medications are also recorded.

Results: As of June 2012, 4349 patients have enrolled in PRISM. Of these, 36.6\% screened positive for potential PBA (CNS-LS score ? 13), including 30.1\% of patients with underlying AD, 46.3\% with ALS, $45.3 \%$ with MS, 36.1\% with stroke, $25.4 \%$ with PD, and 52.9\% with TBI. Average ratings of QOL due to their neurological condition were worse for patients with CNS-LS 13 (6.7 on a 10 point scale) compared with patients with CNS-LS $<13$ (4.6). Patients with CNS-LS ?13 were more likely to be taking antidepressants (SSRIs $39.7 \%$ vs $27.2 \%$, TCAs $20.0 \%$ vs $10.1 \%)$ or antipsychotics (5.3\% vs $2.9 \%$ ) compared with patients with CNS-LS $<13$. A screening for depression or other mood disorders was not conducted.

Conclusions: The PRISM Registry provides novel insight into prevalence of PBA symptoms by prospectively evaluating a large number of patients across diagnoses and clinical settings. The insights gained from this US-based, noninterventional ("naturalistic") strategy for examining PBA prevalence are intriguing and additional longitudinal or non-US epidemiologic registries should be considered.

Educational objectives: Improve participant's awareness and understanding of PBA and its estimated prevalence, as well as the utility of patient registries in epidemiologic research. Understand the utility of the CNS-LS as a screening tool for PBA symptoms.

Funding: Avanir Pharmaceuticals, Inc.

PS-109

A Responder Analysis of Two Approaches for Switching to Iloperidone in Patients With Schizophrenia: Results From the i-FANS Study

Greg Mattingly, MD; Farid Kianifard, PhD; Xiangyi Meng, PhD; Adam K. Winseck, PhD; Marla Hochfeld, MD

\begin{abstract}
:
Study Objective: The objective was to evaluate 2 strategies for switching to iloperidone from other antipsychotics in patients with schizophrenia experiencing suboptimal efficacy and/or safety/tolerability symptoms with their current treatment. A responder analysis evaluated the effect of dose on the Integrated Clinical Global Impression of Change (I-CGI-C; primary variable).

Methods: Subjects in this 12-week, randomized, multicenter, open-label study were adult (18-64 years) outpatients with a DSM-IV-TR diagnosis of schizophrenia who were maintained on risperidone, olanzapine, or aripiprazole and were experiencing suboptimal efficacy or 1 or more predefined tolerability problems. Patients in each of these 3 cohorts were randomized 1:1 to switch immediately to iloperidone or to gradually taper their prior antipsychotic dose (to $50 \%$ on Day 1, 25\% at Week 1, and $0 \%$ at Week 2 ) over the first 2 weeks of iloperidone use. For all subjects, iloperidone was titrated to a target dose of $6 \mathrm{mg}$ twice daily (bid) by Day 4 , followed by increases (no more than $4 \mathrm{mg} / \mathrm{d}$ ) up to $12 \mathrm{mg}$ bid, during Weeks 2-12 based on investigator judgment. For the responder analysis, percentage of responders was summarized by visit and dosage range ( $\leqslant 12-16 \mathrm{mg} / \mathrm{d}$ and $20-24 \mathrm{mg} / \mathrm{d})$, with a responder defined as having an I-CGI-C value $<4$. The I-CGI-C is scored as 1 (very much improved), 2 (much improved), 3 (minimally improved), 4 (no change), 5 (minimally worse), 6 (much worse), or 7 (very much worse).
\end{abstract}


Results: The full analysis set included 500 subjects (240 randomized to gradual switch and 260 to immediate switch) and 346 completed the study (168 in the gradual- and 178 in the immediate-switch groups). Subjects were (mean \pm standard deviation) $43.3 \pm 11.0$ years of age; $56.5 \%$ were Black/African American, and $67 \%$ were men. For all patients (all iloperidone doses $12-24 \mathrm{mg} / \mathrm{d}$ ), the proportion with shifts from normal to high levels in fasting metabolic measures from baseline to Week 12 were similar for the gradual- and immediate-switch groups for serum glucose $(<100 \mathrm{mg} / \mathrm{dL}$ to $\geqslant 126 \mathrm{mg} / \mathrm{dL} ; 11.1 \%$ and $7.5 \%$, respectively), total cholesterol $(<200 \mathrm{mg} / \mathrm{dL}$ to $\geqslant 240 \mathrm{mg} / \mathrm{dL} ; 1.6 \%$ and $2.9 \%$ ), low-density lipoprotein (LDL; $<100 \mathrm{mg} / \mathrm{dL}$ to $\geqslant 160 \mathrm{mg} / \mathrm{dL} ; 0 \%$ and $1.4 \%$ ), and triglycerides $(<150 \mathrm{mg} / \mathrm{dL}$ to $\geqslant 200 \mathrm{mg} / \mathrm{dL} ; 8.8 \%$ and $8.8 \%)$. Shifts from normal to low levels for fasting highdensity lipoprotein (HDL; $\geqslant 40 \mathrm{mg} / \mathrm{dL}$ to $<40 \mathrm{mg} / \mathrm{dL}$ ) were $16.9 \%$ and $7.4 \%$. Respective mean changes from baseline to Week 12 in these fasting measures for gradual- and immediate-switch groups were serum glucose, $7.7 \mathrm{mg} / \mathrm{dL}$ and $7.5 \mathrm{mg} / \mathrm{dL}$; cholesterol, $-0.4 \mathrm{mg} / \mathrm{dL}$ and $-4.3 \mathrm{mg} / \mathrm{dL} ; \mathrm{LDL},-3.2 \mathrm{mg} / \mathrm{dL}$ and $-1.0 \mathrm{mg} / \mathrm{dL} ; \mathrm{HDL}$, $-0.8 \mathrm{mg} / \mathrm{dL}$ and $-0.6 \mathrm{mg} / \mathrm{dL}$; and triglycerides, $14.8 \mathrm{mg} / \mathrm{dL}$ and $-6.3 \mathrm{mg} / \mathrm{dL}$. Mean increases in weight were $0.9 \mathrm{~kg}$ and $0.8 \mathrm{~kg}$, respectively, with $9.6 \%$ and $7.7 \%$ of patients experiencing $\mathrm{a} \geqslant 7 \%$ weight increase over the course of the study.

Conclusions: At Week 12, iloperidone response rates were similar regardless of switch approach or iloperidone dose. Between $76 \%$ and $86 \%$ of the patients responded over the 12 weeks of the study.

Educational Objective: Participant should be able to explain the role of switch approach and iloperidone dose on iloperidone response rates in patients with schizophrenia.

Funding: Novartis Pharmaceuticals Corporation.

PS-110

Metabolic Shifts Following a Switch From Risperidone, Olanzapine, or Aripiprazole to Iloperidone in Patients With Schizophrenia (i-FANS Study)

Greg Mattingly, MD; Xiangyi Meng, PhD; Farid Kianifard, PhD; Adam K. Winseck, PhD; Marla Hochfeld, MD

\begin{abstract}
:
Study Objective: The objective was to evaluate the impact of 2 strategies for switching to iloperidone from risperidone, olanzapine, or aripiprazole in patients with schizophrenia experiencing suboptimal efficacy and/or safety/tolerability symptoms on their current treatment.

Methods: This was a 12-week randomized, multicenter, open-label study. Subjects were adult (18-64 years) outpatients with a DSM-IV-TR diagnosis of schizophrenia who were experiencing suboptimal efficacy or 1 or more predefined tolerability problems on their current treatment of risperidone, olanzapine, or aripiprazole. Patients were randomized 1:1 to switch immediately to iloperidone or to gradually taper their prior antipsychotic dose (to $50 \%$ on Day 1, 25\% at Week 1, and $0 \%$ at Week 2 ) over the first 2 weeks of iloperidone use. For all patients, iloperidone was titrated to a target dose of $6 \mathrm{mg}$ twice daily (bid) by Day 4, which could be increased up to $12 \mathrm{mg}$ bid based on investigator judgment. The primary variable was the Integrated Clinical Global Impression of Change. Metabolic measures were obtained under fasting conditions. Metabolic analyses were conducted for the 3 cohorts combined.
\end{abstract}

Results: The full analysis set included 500 subjects (240 randomized to gradual switch and 260 to immediate switch) and 346 completed the study (168 in the gradual- and 178 in the immediate-switch groups). Subjects were (mean \pm standard deviation) $43.3 \pm 11.0$ years of age; $56.5 \%$ were Black/African American, and $67 \%$ were men. For all patients (all iloperidone doses $12-24 \mathrm{mg} / \mathrm{d}$ ), the proportion with shifts from normal to high levels in fasting metabolic measures from baseline to Week 12 were similar for the gradual- and immediate-switch groups for serum glucose $(<100 \mathrm{mg} / \mathrm{dL}$ to $\geqslant 126 \mathrm{mg} / \mathrm{dL} ; 11.1 \%$ and $7.5 \%$, respectively), total cholesterol $(<200 \mathrm{mg} / \mathrm{dL}$ to $\geqslant 240 \mathrm{mg} / \mathrm{dL} ; 1.6 \%$ and $2.9 \%$ ), low-density lipoprotein (LDL; $<100 \mathrm{mg} / \mathrm{dL}$ to $\geqslant 160 \mathrm{mg} / \mathrm{dL} ; 0 \%$ and $1.4 \%$ ), and triglycerides $(<150 \mathrm{mg} / \mathrm{dL}$ to $\geqslant 200 \mathrm{mg} / \mathrm{dL} ; 8.8 \%$ and $8.8 \%)$. Shifts from normal to low levels for fasting highdensity lipoprotein (HDL; $\geqslant 40 \mathrm{mg} / \mathrm{dL}$ to $<40 \mathrm{mg} / \mathrm{dL}$ ) were $16.9 \%$ and $7.4 \%$. Respective mean changes from baseline to Week 12 in these fasting measures for gradual- and immediate-switch groups were serum glucose, $7.7 \mathrm{mg} / \mathrm{dL}$ and $7.5 \mathrm{mg} / \mathrm{dL}$; cholesterol, $-0.4 \mathrm{mg} / \mathrm{dL}$ and $-4.3 \mathrm{mg} / \mathrm{dL} ; \mathrm{LDL},-3.2 \mathrm{mg} / \mathrm{dL}$ and $-1.0 \mathrm{mg} / \mathrm{dL} ; \mathrm{HDL}$, $-0.8 \mathrm{mg} / \mathrm{dL}$ and $-0.6 \mathrm{mg} / \mathrm{dL}$; and triglycerides, $14.8 \mathrm{mg} / \mathrm{dL}$ and $-6.3 \mathrm{mg} / \mathrm{dL}$. Mean increases in weight were $0.9 \mathrm{~kg}$ and $0.8 \mathrm{~kg}$, respectively, with $9.6 \%$ and $7.7 \%$ of patients experiencing a $\geqslant 7 \%$ weight increase over the course of the study.

Conclusions: The proportions of patients who experienced metabolic shifts following a switch to iloperidone from risperidone, olanzapine, or aripiprazole were between $0 \%$ and $16.9 \%$, depending on the metabolic measure, and were consistent with previously reported data (Fanapt $\mathbb{R}$ [iloperidone] prescribing information). 
Educational Objective: Participant should be able to explain the frequency of metabolic shifts following a gradual or immediate switch from risperidone, olanzapine, or aripiprazole to iloperidone in patients with schizophrenia.

Funding: Novartis Pharmaceuticals Corporation.

\title{
PS-111
}

Trajectory of Improvement in Clinical Outcomes Following a Gradual or Immediate Switch to Iloperidone in Patients With Schizophrenia: The i-FANS Study

Gustavo Alva, MD, DFAPA; Farid Kianifard, PhD; Xiangyi Meng, PhD; Adam K. Winseck, PhD; Marla Hochfeld, MD

\begin{abstract}
:
Study Objective: The objective was to evaluate clinical outcomes with iloperidone following 2 switching approaches (gradual or immediate) in adults with schizophrenia exhibiting suboptimal efficacy and/or safety/ tolerability from their current antipsychotic treatment of risperidone, olanzapine, or aripiprazole.
\end{abstract}

Methods: In this 12-week randomized, multicenter, open-label study, patients with schizophrenia who were experiencing inadequate efficacy and/or poor tolerability on current treatment with risperidone, olanzapine, or aripiprazole were randomized to switch to iloperidone either by an immediate discontinuation of current treatment at baseline (BL) or a gradual discontinuation by reducing current treatment to $50 \%$ on Day $1,25 \%$ at Week 1 , and $0 \%$ at Week 2. Patients received iloperidone $1 \mathrm{mg}$ twice daily (bid) on Day 1 titrated over 4 days to $6 \mathrm{mg}$ bid, followed by increases (no more than $4 \mathrm{mg} / \mathrm{d}$ ) up to $12 \mathrm{mg}$ bid, if needed, during Weeks $2-12$. The primary variable was the Integrated Clinical Global Impression of Change (I-CGI-C), an integrated CGI-C score ranging from 1 (very much improved) to 7 (very much worse), and the primary analysis time point was Week 12. Secondary variables were changes from BL in Efficacy CGI of Severity (E-CGI-S), Safety and Tolerability CGI of Severity (ST-CGI-S), and Integrated CGI of Severity (I-CGI-S), which are each scored from 1 (absence of symptoms) to 7 (most severe).

Results: Of the 500 randomized subjects (260 to immediate and 240 to gradual switch), 346 completed the study (168 in the gradual- and 178 in the immediate-switch groups). Respective discontinuation rates in the gradual- and immediate-switch groups were $25.9 \%$ and $25.5 \%$ for risperidone; $36.7 \%$ and $36.8 \%$ for olanzapine, and $27.5 \%$ and $33.3 \%$ for aripiprazole. Subjects were (mean \pm standard deviation) $43.3 \pm 11.0$ years of age; $56.5 \%$ were Black/ African American, and 67\% were men. In the 3 cohorts combined, least-squares means (LSM) at Week 12 were similar between the gradual- and immediate-switch approaches for the I-CGI-C (2.826 [gradual]; 2.837 [immediate]). LSM changes from BL to Week 12 were similar between switch approaches for the E-CGI-S $(-0.831$ [gradual] and -0.825 [immediate]), ST-CGI-S $(-0.888$ and -0.802$)$, and I-CGI-S $(-0.887$ and -0.879$)$. Improvement was relatively steady throughout the study for both gradual- and immediate-switch groups for the I-CGI-C, E-CGI-S, ST-CGI-S, and I-CGI-S scores starting at Week 1 and continuing weekly through Week 12. For the 3 treatment cohorts combined, differences in scores between treatment groups were not clinically meaningful at any time point for any of these endpoints. For the individual cohorts, improvements were similar and differences in scores between treatment groups were generally not clinically meaningful.

Conclusions: Overall, the pattern of clinical improvement in patients with schizophrenia was similar regardless of iloperidone switch approach. Improvement was seen at Week 1 and continued throughout the 12 weeks of the study.

Educational Objective: Participant should be able to discuss the effect of a gradual or immediate switch from risperidone, olanzapine, or aripiprazole to iloperidone on the pattern of clinical improvement in patients with schizophrenia.

Funding: Novartis Pharmaceuticals Corporation.

\section{PS-112}

Antidepressant medications modulate cellular cytokine production in depressed subjects

Sirid Kellermann, PhD, MBA; Deanna J. Fall, B.A.; David T Marc, MBS

\begin{abstract}
:
Study Objective: This study aimed to assess the effects of antidepressant medications on immune function by measuring white blood cell cytokine production.
\end{abstract}

Method: We studied depressed subjects who were either not taking any medication $(\mathrm{n}=85)$, or taking antidepressant medications $(n=49)$, as well as non-depressed controls $(n=131)$. Depression was defined by relevant ICD-9 codes assigned by subjects' healthcare practitioners. Non-depressed subjects were not diagnosed 
with depression or any other mental disorder. Medication status was determined from the patients' reported medical history. Medications included selective serotonin reuptake inhibitors (SSRI; $n=23$ ), serotoninnorepinephrine inhibitors (SNRI; $n=18)$, a combination of SSRI and SNRI $(n=3)$, or tricyclic agents $(n=5)$.

Peripheral blood mononuclear cells (PBMC) were isolated from subjects and cultured for 24 hours with or without stimulants (lipopolysaccharide (LPS) or phytohemagglutinin (PHA)), after which the supernatants were collected and cytokines measured via a multiplex approach. Differences between cytokine levels were determined using one-way ANOVA.

Results: PBMC from depressed patients taking antidepressants had lower production of several pro-inflammatory cytokines (IFN-gamma ( $p<0.01)$, IL-8 ( $<<0.01)$, MCP-1 ( $p=0.026)$, and MIP-1beta $(p=0.029)$ ) compared to those from untreated depressed patients. However, we observed elevations in the Th1 cytokine IL-12 ( $p<0.01)$ as well as the Th17 cytokine IL-17 ( $p<0.01)$. Production of the Th2 cytokine IL-13 $(p<0.01)$ was also higher in medicated patients, as was IL-7 ( $\mathrm{p}<0.01)$ and G-CSF ( $\mathrm{p}=0.047)$. IL-2 and TNF-alpha were elevated, but did not reach statistical significance. There were no significant differences in IL-1beta, IL-2, IL-6, and IL-10.

Besides being able to directly monitor cytokine production by PBMC, our method additionally allowed us to evaluate the effects of PHA and/or LPS stimulation. By increasing cytokine secretion, these agents amplified differences between patient cohorts for certain cytokines such as IFN-gamma, IL-12, and IL-17 that would not otherwise have reached statistical significance.

Conclusions: Antidepressant medication is associated with heterogeneous effects on PBMC cytokine expression. We identified two novel biomarkers, IL-13 and IL-7 that were elevated with medication. IL-13 may attenuate macrophage cytokine production, while IL-7 may aid in immune recovery and a return to immune homeostasis. Compared to serum cytokine assessment, measuring PBMC cytokine production may provide more direct insights into the effects of antidepressants on immune function at a cellular level. Additionally, PBMC stimulation can be valuable in revealing modulations in cytokine expression that might otherwise be underappreciated in unstimulated PBMC or in serum.

Educational Objective(s): These results reflect the most comprehensive assessment to date of cytokine alterations associated with the administration of antidepressants in clinical depression. This material provides a greater appreciation of the complex effects of antidepressant medications on the immune system which transcend simplistic Th1/Th2 and/or inflammatory/anti-inflammatory paradigms.

Funding: Funding provided by NeuroScience, Inc.

\title{
PS-113
}

Effect of L-methylfolate on Core Symptoms in a Randomized Clinical Trial of Patients with Major Depression Richard C. Shelton, MD; George I. Papakostas, MD; Maurizio Fava, MD; Stephen M. Stahl, MD, PhD

\begin{abstract}
:
Background: Major depressive disorder (MDD) includes a metabolic component that is associated with poor antidepressant response, and correction may improve core symptoms. This analysis assessed the effect of L-methylfolate $15 \mathrm{mg}$ as an adjunct to SSRIs on core symptoms of depression and correlations with other biomarkers.
\end{abstract}

Methods: 75 inadequate responders to SSRIs were enrolled in a 60-day, multi-center, double-blind, placebocontrolled trial. Patients received L-methylfolate $15 \mathrm{mg}$ /day for 60 days, placebo for 30 days followed by L-methylfolate $15 \mathrm{mg}$ /day for 30 days, or placebo for 60 days. In a sub-analysis study of the effects on core symptoms of depression, mean change from baseline to endpoint was evaluated for the Maier subscale (HDRS items 1, 2, 7-10, and 13) for L-methylfolate and placebo. In addition, correlations between BMI and other biomarkers were examined.

Results: 74 patients were enrolled. For pooled data, mean change on the Maier subscale was $-3.3 \pm 3.7$ for L-methylfolate vs. $-1.5 \pm 3.2$ for placebo $(95 \%$ CI: $-2.936,-0.296, p=0.016)$. Mean improvement in core symptoms of depression was significantly greater with L-methylfolate vs. placebo among patients with a BMI $? 30 \mathrm{~kg} / \mathrm{m}^{2}$ (phase-pooled difference in mean change: $-2.637 ; 95 \% \mathrm{CI}:-4.410,-0.864$ ).

Conclusions: A robust response in core symptoms of depression on the Maier subscale was observed with L-methylfolate as an adjunct to SSRIs. Improvement in core symptoms of depression could be associated with correction of the underlying metabolic imbalances involved in depression. 


\title{
PS-114
}

Varenicline for Smoking Cessation: From Clinical Trials to Efficacy Data

Chaitanya Pabbati, MD; Isabel Domingues, MD

\begin{abstract}
:
Study Objectives: To understand the role, efficacy, and feasibility of utilizing varenicline as a successful agent in smoking cessation.

Introduction: Cigarette smoking is one of the principal causes of preventable morbidity and mortality in the developed world, responsible for an estimated 435,000 premature deaths in the United States. Nicotine dependence itself is a chronic, relapsing addiction affecting more than $20 \%$ of the population worldwide. Adequate pharmacotherapy can at least double quit rates over counseling alone, and seven first-line pharmacologic treatments are currently available, with varenicline being one of the latest agents. The goals of this project are to review scientific evidence of the mechanism of action of varenicline, the efficacy of its treatment of nicotine addiction, and to tie this information together by presenting the results of an efficacy study carried out at a community-based smoking cessation clinic.
\end{abstract}

Mechanism of Action: Varenicline's efficacy for smoking cessation is due to the attenuation of rewarding response and heart rate induced by nicotine. In mice models, nicotine has been shown to act as an agonist at beta2 nicotinic acetylcholine receptors (beta2-nAChR), leading to locomotor depression and a decrease in core body temperature. Varenicline however acts on the alpha4-beta2-nAChR, though only as a partial agonist, or a functional antagonist, so as to not produce the effects from nicotine binding. Partial stimulation such as this does not lead to a corresponding release in dopamine from the mesolimbic and mesocortical reward pathways. In addition to this, varenicline also blocks the ability of nicotine to bind and lead to dopamine release. Varenicline's effects on the 5-HT3 receptor as an agonist are thought to attribute for it's side effects, namely those of altered mood or suicidal ideation.

Prior Clinical Trials: Studies have shown that varenicline increases the odds of successful smoking cessation two to three times more efficaciously than with pharmacologically unassisted quit attempts. With a relative risk as compared to placebo of 2.38 (95\% CI 2.00, 2.84), varenicline has been found to be one of the most effective pharmacotherapies. Indeed, more participants quit successfully with varenicline than with bupropion (RR 1.52 [95\% CI 1.22, 1.88]) or nicotine replacement (RR 1.31 [95\% CI 1.01, 1.71]). Additionally, there is some evidence suggesting that varenicline may have a role to play in relapse prevention. The recommended dosage of varenicline is $1 \mathrm{mg}$ twice daily, and the main adverse effect is nausea. Possible serious adverse events, including depressed mood, agitation and suicidal thoughts, have also been reported.

Methods: A Study in a Community Smoking Cessation Clinic

345 patients enrolled in a free community smoking cessation clinic were studied to determine the feasibility of using varenicline for smoking cessation. Each patient was enrolled in a 12-week program in which they either received varenicline, nicotine replacement therapy (NRT), and weekly counseling, or NRT and counseling alone if they had a contraindication to treatment with varenicline therapy. Contraindications and general medical status were determined at the index treatment session which lasted one hour and included a comprehensive review of systems, while subsequent sessions were 15-20 minutes in length. Each week, patients attended the clinic at a designated time and met with a trained smoking cessation counselor with whom they discussed their cigarette usage. Patients were also given a breath carbon monoxide $(\mathrm{CO})$ test to determine levels of $\mathrm{CO}$ in their breath, and also as a way to demonstrate the efficacy of the treatment for the patient. Finally, each patient was assessed for adverse side effects. By the end of the study, 289 patients had attended a minimum of 3 of the 12 sessions. The remainder of patients were either lost to follow-up $(\mathrm{N}=12)$, left the program before 3 weeks was completed $(\mathrm{N}=31)$, or had a side effect warranting a change in therapy $(\mathrm{N}=13)$. The 289 remaining patients were then phoned one month after their scheduled 12th week to determine the status of their smoking and nicotine intake. Cessation was defined as no cigarette intake at one month following the 12th week as per patient report, and supported by a reading of 0.0 on the CO monitor.

Results: $67.5 \%$ of patients $(\mathrm{N}=195)$ received varenicline therapy, of whom $68.7 \%$ were self-reported non-smokers 1-month after the 12 week study period had ended, as compared to $31.9 \%(\mathrm{~N}=30)$ of the patients who received $\mathrm{NRT}$, leading to a $\mathrm{p}<0.0001$. Factoring in compliance, $76.9 \%(\mathrm{~N}=150)$ of all patients receiving varenicline attended 4 or more of the 12 week sessions. Of these, $86.7 \%$ were self-reported non-smokers 1 -month after the 12 week study period had ended, as compared to $40.7 \%(\mathrm{~N}=22)$ of the 54 patients receiving NRT and counseling alone who attended 4 or more sessions. This also is associated with a p-value less than 0.0001 . Further calculation yields a number needed to treat (NNT) of 3, with an absolute risk reduction (ARR) of $45.93 \%$.

Conclusions: A nicotine partial agonist FDA approved since 2006, Varenicline is currently considered the most efficacious pharmacotherapy for smoking cessation. Our data obtained through a controlled trial conducted at a free community smoking cessation clinic supports most of the clinical trials found in the literature, showing supremacy of varenicline 
when compared to NRT plus counseling (ARR of 45.93\%). Further varenicline trials are needed in order to assess maintenance of nicotine abstinence in the long term. Additional studies would also be needed to determine varenicline's efficacy as compared to other methods of smoking cessation, such as treatment with bupropion. However, given its efficacy compared to NRT alone, it is clear the varenicline has an important role to play in community smoking cessation.

Educational Objective: After studying this material, the reader should be able to understand the fundamentals of varenicline as an agent in the treatment of nicotine dependence, describe the basic mechanism of action of varenicline as a alpha4 beta2-nAChR partial agonist, and explain the efficacy and utility of varenicline as a treatment option for smoking cessation.

Funding: No funding

\title{
PS-115
}

Switching from olanzapine to lurasidone: results from a 6-month open label extension study

Stephen M. Stahl, MD, PhD; Vamsi K. Bollu, PhD; Krithika Rajagopalan, PhD; Andrei Pikalov, MD, PhD;

Jay Hsu, PhD; Antony Loebel, MD

\begin{abstract}
:
Study Objectives: In recent longer-term treatment studies of schizophrenia, all-cause treatment discontinuation has been used as a clinically meaningful composite measure of treatment efficacy, safety and tolerability. The aim of this analysis of a 6-month lurasidone open-label extension (OLE) study was to compare the efficacy, safety, and allcause discontinuations among patients switching to lurasidone from olanzapine or placebo vs. patients continuing on lurasidone during a 6 month OLE study.
\end{abstract}

Method: The core study for this 6-month OLE was a 6-week, double-blind (DB), placebo-controlled trial of lurasidone $40 \mathrm{mg}$ and $120 \mathrm{mg}$ fixed doses, with olanzapine $15 \mathrm{mg}$ as an active-treatment arm (for assay sensitivity). Eligible patients continuing into the OLE received lurasidone fixed dose $(80 \mathrm{mg})$ for 1-week followed by flexible dose (40-120 mg). Assessments included for analysis were: efficacy (PANSS total score changes); safety (changes in cardio-metabolic parameters), rates of all-cause discontinuation and reasons for discontinuation.

Results: From 254 patients in the OLE, 118 patients continued on lurasidone, 71 and 65 patients switched from olanzapine or placebo to lurasidone, respectively. Mean total PANSS scores decreased from 66.6 (OLE baseline) to 54.9 (OC analysis of OLE endpoint); with similar PANSS score improvements seen among those switching to lurasidone and those continuing on lurasidone. While no significant weight changes were observed among lurasidone patients during DB or when they continued on lurasidone in OLE, patients switching from olanzapine to lurasidone showed significant and sustained weight loss during OLE. Improvements in lipids and other cardiometabolic parameters were also observed among patients switching from olanzapine to lurasidone. Total all-cause discontinuations were $55.5 \%(n=141 / 254)$ at OLE endpoint; with similar rates of discontinuation between those who stayed on lurasidone $(53.4 \%)$ and those switching from olanzapine $(56.3 \%)$ or placebo (58.5\%), respectively. Discontinuation rates were also approximately similar among patients staying on lurasidone or switching from olanzapine or placebo, respectively, due to lack of efficacy $(7.6 \%, 7.0 \%, 4.6 \%)$ and adverse effects $(14.4 \%, 9.9 \%, 12.3 \%)$.

Conclusions: In this analysis, patients with schizophrenia who switched from olanzapine (DB phase) to lurasidone (OLE) maintained efficacy improvements at 6-months and experienced similar rates of all-cause discontinuations as those who continued on lurasidone. In addition, significant weight loss and improved lipid and other cardiometabolic parameters were observed after switch to lurasidone.

Educational Objectives: After reviewing this poster, the participant may be better able to understand the longer-term safety and efficacy of lurasidone in patients with schizophrenia. In addition, they may also be able to understand the effect of switching treatment from olanzapine to lurasidone.

Funded by Sunovion Pharmaceuticals Inc.

\section{PS-116}

Effect of 12 months of lurasidone on weight in subjects with schizophrenia

Andrei Pikalov, MD, PhD; Jonathan M. Meyer, MD; Yongcai Mao, PhD; Josephine Cucchiaro, PhD;

Antony Loebel, MD

\section{Abstract:}

Study Objectives: Individuals with schizophrenia have an increased prevalence of obesity (Newcomer et al, 2005). Furthermore, notable differences have been reported among atypical antipsychotics in effects on weight. 
This post-hoc analysis was conducted to evaluate the effect of 12 months of treatment with lurasidone on weight and body mass index (BMI) in subjects with schizophrenia.

Method: A post-hoc, observed case (OC) analysis was performed on pooled data from 6 clinical studies that evaluated the safety of 12 months of treatment with lurasidone (40-120 mg/day).

Results: The analysis sample consisted of 371 subjects who completed 12 months of treatment with lurasidone (mean age, 42.4 years; male, 66.6\%; white, 31.0\%; black, 24.8\%, Asian, $40.2 \%$; other, $4.0 \%$ ). The mean (SD) weight at baseline was $74.0(19.1) \mathrm{kg}$ and the mean BMI was $25.9(5.3) \mathrm{kg} / \mathrm{m}^{2}$, with $3.2 \%$ of subjects meeting standard BMI criteria for being underweight, $48.8 \%$ normal weight, $26.4 \%$ overweight, and $21.6 \%$ obese. On an OC analysis, the mean change in weight was $-0.74 \mathrm{~kg}$ at 3 months, $-0.65 \mathrm{~kg}$ at 6 months, and $-0.71 \mathrm{~kg}$ at 12 months. The mean change in BMI was $-0.26 \mathrm{~kg} / \mathrm{m}^{2}$ at 3 months, $-0.22 \mathrm{~kg} / \mathrm{m}^{2}$ at 6 months, and $-0.24 \mathrm{~kg} / \mathrm{m}^{2}$ at 12 months. An increase of $7 \%$ in weight occurred in $2.7 \%$ of subjects at 3 months, $8.6 \%$ at 6 months, and $17.6 \%$ at 12 months. An decrease of $7 \%$ in weight occurred in $9.7 \%$ of subjects at 3 months, $17.1 \%$ at 6 months, and $22.2 \%$ at 12 months. Overall, $12.0 \%$ of subjects shifted, by month 12 , from the underweight/normal BMI category at baseline to the overweight category, and none to obese. Conversely, $28.6 \%$ of subjects shifted, by month 12 , from overweight to normal weight.

Conclusions: The results of this pooled analysis of subjects who completed 12 months of treatment suggest that lurasidone, in the dosing range of $40-120 \mathrm{mg}$, was associated with a low potential for clinically significant weight gain.

Educational Objectives: After reviewing this poster, the participant may be better able to understand the potential impact of 12 months of treatment with lurasidone on weight in patients diagnosed with schizophrenia.

Sponsored by Sunovion Pharmaceuticals Inc.

PS-117

Switching to lurasidone in patients with schizophrenia: tolerability and effectiveness of three switch strategies Joseph P. McEvoy, MD; Leslie Citrome, MD, MPH; David Hernandez, BA; Jay Hsu, PhD; Andrei Pikalov, MD, PhD; Josephine Cucchiaro, PhD; Antony Loebel, MD

\begin{abstract}
:
Study Objectives: To evaluate the safety, tolerability and effectiveness of switching clinically stable, but symptomatic non-acute patients with schizophrenia or schizoaffective disorder to lurasidone.

Method: Non-acute patients who met DSM-IV criteria for schizophrenia or schizoaffective disorder and who were considered to be appropriate candidates for switching current antipsychotic medication to lurasidone, were randomized to three switch strategies: a $40 / 40$ group $(\mathrm{N}=74)$ was started on a dose of $40 \mathrm{mg} / \mathrm{d}$ for 2 weeks; a $40 /$ 80 group $(\mathrm{N}=88)$ was started on a dose of $40 \mathrm{mg} / \mathrm{d}$ for 7 days, then increased to $80 \mathrm{mg} / \mathrm{d}$ for 7 days; and an $80 / 80$ group $(\mathrm{N}=82)$ was started on a dose of $80 \mathrm{mg} / \mathrm{d}$ for 14 days. All patients were then treated for an additional 4 weeks with lurasidone $40-120 \mathrm{mg} / \mathrm{d}$, flexibly dosed. The prior antipsychotic agent was tapered and discontinued over the initial 2-week study period. Patients were stratified based on whether the primary pre-switch antipsychotic was sedating (olanzapine, quetiapine) or non-sedating (all others). Time to treatment failure was evaluated, defined as insufficient clinical response, exacerbation of underlying disease or discontinuation due to an adverse event (AE). Safety parameters were also assessed.
\end{abstract}

Results: Switching to lurasidone was well-tolerated with $81.1 \%$ completing the 6-week study. No clinically relevant differences in efficacy or tolerability were noted when comparing the 3 different switch strategies. Time to treatment failure numerically differed among patients who had been receiving a sedating antipsychotic $(35.8 \%$ of the total) immediately prior to the switch to lurasidone compared to those who were receiving a non-sedating antipsychotic $(\log$ rank $\mathrm{p}=0.101)$, with a treatment failure rate of $11.6 \% \mathrm{vs} .5 .8 \%$, respectively. Treatment with lurasidone was associated with LS mean within-group improvement at endpoint on the PANSS of -5.8 (95\%-CI, $-7.0,-4.5$; Cohen's $\mathrm{d}, 0.5)$. For the total sample, treatment with lurasidone was associated with $-0.3 \mathrm{~kg}$ mean decrease in weight, and a median reduction in both cholesterol $(-1.0 \mathrm{mg} / \mathrm{dL})$ and triglycerides $(-6.0 \mathrm{mg} / \mathrm{dL})$.

Conclusions: In this study, switching to lurasidone was well-tolerated using a cross taper strategy regardless of initial dose used or rate of titration. Patients switching to lurasidone demonstrated clinically relevant improvement in efficacy measures. Overall reductions in weight, lipids and glucose were observed, and the AE profile was similar to previous lurasidone studies.

Educational Objectives: After reviewing this poster, the participant may be better able to understand the potential safety and efficacy of lurasidone in patients diagnosed with schizophrenia when considering a switch from their previous antipsychotic treatment. 
Trial Registration: clinicaltrials.gov identifier: NCT01143077

Sponsored by Sunovion Pharmaceuticals Inc.

PS-118

Effectiveness of lurasidone vs. quetiapine XR for relapse prevention in schizophrenia: a 12-month, double-blind study

Antony Loebel, MD; Josephine Cucchiaro, PhD; Jane Xu, PhD; Kaushik Sarma, MD; Andrei Pikalov, MD, PhD; John M. Kane, MD

Abstract:

Study Objectives: To evaluate the efficacy and safety of lurasidone vs. quetiapine XR (QXR) in preventing relapse in subjects with chronic schizophrenia.

Method: After completing an initial double-blind, placebo-controlled, 6 week trial with fixed doses of lurasidone $(80 \mathrm{mg} ; 160 \mathrm{mg})$ or QXR $(600 \mathrm{mg})$, subjects received 12 months of double-blind, flexible once-daily doses of lurasidone $(40-160 \mathrm{mg})$ vs. QXR $(200-800 \mathrm{mg})$. The primary a priori efficacy comparison was between subjects treated with lurasidone $(n=139)$ and QXR $(n=79)$ who were clinical responders after acute treatment. The primary endpoint, time-to-relapse, was analyzed using a Cox proportional hazards model, with a pre-specified non-inferiority margin for the risk of relapse hazard ratio of 1.93 .

Results: Lurasidone was non-inferior to QXR in risk for relapse over the 12 month treatment period (hazard ratio 0.728 , $95 \%$ CI $[0.410,1.295])$. The risk of relapse in lurasidone treated subjects was reduced by $27.2 \%$ (hazard ratio 0.728 ) compared with QXR. The Kaplan-Meier estimate of the probability of relapse at 12 months was lower for lurasidone vs. QXR (0.237 vs. 0.336). Treatment with lurasidone (modal daily dose $120 \mathrm{mg}$ ) was associated with a significantly greater change in PANSS total scores compared with QXR (modal dose $600 \mathrm{mg}$ ) on an MMRM analysis. Rates of adverse events ?5\% in the lurasidone group were akathisia $(12.6 \%)$, headache $(10.6 \%)$, insomnia $(7.9 \%)$, anxiety $(6.0 \%)$, parkinsonism $(6.0 \%)$, and weight increased (6.0\%). Analysis of changes from acute study baseline to 12 months of treatment (OC) with lurasidone and QXR, respectively, showed a mean change in weight of $+0.7 \mathrm{vs.}+1.2 \mathrm{~kg}$; a median change in glucose of +1.0 vs. $+1.0 \mathrm{mg} / \mathrm{dL}$; a median change in cholesterol of $0.0 \mathrm{vs}$. $+4.0 \mathrm{mg} / \mathrm{dL}$; and a median change in triglycerides of -18.0 vs. $-7.0 \mathrm{mg} / \mathrm{dL}$. There were no clinically meaningful changes in other laboratory or ECG parameters on either drug.

Conclusions: This long-term, double-blind study demonstrated non-inferiority of lurasidone to QXR in prevention of relapse over a 12 month period, with a $27.2 \%$ reduction in relapse risk compared with QXR. Treatment with lurasidone was associated with few adverse effects on metabolic parameters, and a minimal effect on weight. The safety and tolerability of lurasidone was consistent with the results of previous studies.

Educational Objectives: After reviewing this poster, the participant should have a better understanding of the efficacy and safety of longer-term treatment of patients diagnosed with schizophrenia with lurasidone or quetiapine XR for the prevention of relapse.

Trial Registration: clinicaltrials.gov identifier: NCT00789698

Sponsored by Sunovion Pharmaceuticals Inc.

\section{PS-119}

Lurasidone monotherapy for the treatment of bipolar I depression: results of a 6-week, double-blind, placebo-controlled study

Antony Loebel, MD; Josephine Cucchiaro, PhD; Robert Silva, PhD; Kaushik Sarma, MD; Jay Hsu, PhD;

Hans Kroger, MS; Joseph Calabrese, MD; Gary Sachs, MD

\footnotetext{
Abstract:

Study Objectives: To evaluate the efficacy and safety of lurasidone, flexibly dosed at 20-60 mg/day or 80-120 mg/ day, in the treatment of major depressive episodes in patients with bipolar I depression without psychotic features.

Method: Subjects meeting DSM-IV-TR criteria for bipolar I depression, with or without rapid cycling, with a Montgomery Asberg Depression Rating Scale (MADRS) score ?20 and a Young Mania Rating Scale score ?12, were randomized to 6 weeks of once-daily, double-blind treatment with either lurasidone 20-60 mg (LUR20-60), lurasidone 80-120 mg (LUR80-120) or placebo (PBO). Primary and key secondary endpoints were change from baseline to week 6 endpoint in MADRS and CGI-bipolar severity (CGI-BP-S) depression scores, respectively, analyzed using mixed model repeated measures (MMRM). Additional secondary outcome measures were analyzed using analysis of covariance, last observation carried forward (ANCOVA-LOCF), or logistic regression.
} 
Results: Study completion rates were 74.1\% in the LUR20-60 group (n/N = 123/166; mean modal dose, $34.9 \mathrm{mg} / \mathrm{d}$ ), $73.4 \%$ in the LUR80-120 group ( $\mathrm{n} / \mathrm{N}=124 / 169$; mean modal dose, $92.3 \mathrm{mg} / \mathrm{d}$ ) and $74.7 \%$ in the PBO group $(\mathrm{n} / \mathrm{N}=127 / 170)$. Lurasidone treatment resulted in significantly greater MADRS score reduction at Week 6 endpoint for both the LUR20-60 group $(-15.4 ; \mathrm{p}<0.001$; effect size $=0.51)$ and the LUR80-120 group ( -15.4 ; $\mathrm{p}<0.001$, effect size $=0.51)$ vs. PBO $(-10.7)$. Both LUR groups separated significantly from PBO from week 2 onward. Lurasidone treatment resulted in significantly greater endpoint reduction in CGI-BP-S depression scores for both the LUR20-60 group $(-1.8 ; \mathrm{p}<0.001)$ and the LUR80-120 group $(-1.7 ; \mathrm{p}<0.001)$ compared with PBO (-1.1). Responder rates (reduction in MADRS ?50\%) were significantly higher for LUR20-60 (53\%) and LUR80-120 (51\%) compared with PBO (30\%; p < 0.001 for both comparisons). Both LUR20-60 and LUR 80-120 groups showed significant improvement vs. PBO on the Hamilton Anxiety Rating scale (p?0.05), the Sheehan Disability Scale (p?0.01), the self-rated Quick Inventory of Depressive Symptomatology (p?0.01), and the Quality of Life, Enjoyment and Satisfaction Questionnaire (p?0.01). Discontinuation rates due to adverse events for LUR20-60 (7\%) and LUR80-120 (6\%) were similar to PBO (6\%). For LUR20-60, LUR80-120, and PBO, respectively, the most frequently reported adverse events were nausea $(10.4 \%, 17.4 \%, 7.7 \%)$, headache $(14.0 \%$, $9.0 \%, 11.9 \%)$, and akathisia $(7.9 \%, 10.8 \%, 2.4 \%)$. Minimal changes in weight, lipids and measures of glycemic control were observed.

Conclusions: In this study, monotherapy with lurasidone, flexibly dosed at 20-60 mg/day or $80-120 \mathrm{mg} /$ day, significantly reduced depressive symptoms in patients with bipolar I depression compared to placebo. Tolerability and safety of lurasidone was consistent with results of previous studies in schizophrenia.

Educational Objectives: After reviewing this poster, the participant may be better able to understand the potential effect of lurasidone as monotherapy on depressive symptoms in patients with bipolar I depression.

The following information concerns a use of lurasidone that has not been approved by the U.S. Food and Drug Administration

Trial registration: clinicaltrials.gov identifier: NCT00868699

Sponsored by Sunovion Pharmaceuticals Inc.

\title{
PS-120
}

Lurasidone adjunctive to lithium or valproate for the treatment of bipolar I depression: results of a 6-week, double-blind, placebo-controlled study

Antony Loebel, MD; Josephine Cucchiaro, PhD; Robert Silva, PhD; Jay Hsu, PhD; Kaushik Sarma, MD;

Gary Sachs, MD; Joseph Calabrese, MD

\begin{abstract}
:
Study Objectives: To evaluate the efficacy and safety of lurasidone, adjunctive to lithium or valproate, in patients with bipolar I depression, without psychotic features.

Method: Subjects meeting DSM-IV-TR criteria for bipolar I depression with a Montgomery Asberg Depression Rating Scale (MADRS) score 20, were randomized to 6 weeks of double-blind (DB) treatment with either lurasidone $20-120 \mathrm{mg}$ /day (LUR) or placebo (PBO), both adjunctive to either lithium (Li) or valproate (VPA). Therapeutic blood levels of Li or VPA had to be maintained for ? 28 days prior to randomization. Changes from DB baseline (DB BL) in MADRS (primary endpoint), and secondary efficacy outcomes were analyzed using either mixed model repeated measures (MMRM) or analysis of covariance, last observation carried forward (ANCOVA-LOCF), or logistic regression.
\end{abstract}

Results: Overall, 78\% of LUR- (143/183) and 83\% of PBO-treated subjects (136/163) completed the study. Mean MADRS scores at DB BL were similar for LUR (30.6) and PBO (30.8), indicative of moderate-to-severe depression. At Week 6 study endpoint, LUR was associated with a significantly greater MADRS reduction vs. PBO (-17.1 vs. -13.5; $\mathrm{p}<0.01 ; \mathrm{MMRM})$. Similarly, LUR treatment reduced CGI-bipolar severity (CGI-BP-S) depression ratings by -2.0 vs. -1.5 for PBO ( $<0.01 ; \mathrm{MMRM}$ ), and improved Sheehan Disability Scale (SDS) scores by -9.5 vs. -7.0 for PBO ( $<<0.05$; ANCOVA-LOCF). Significant improvements vs. PBO were also observed for anxiety symptoms, assessed by the HAM-A total score ( -8.0 vs. $-6.0 ; \mathrm{p}<0.01$; ANCOVA-LOCF), and in quality of life, assessed by the Quality of Life, Enjoyment and Satisfaction Questionnaire (Q-LES-Q-SF; +22.2 vs. +15.9; p < 0.01; ANCOVALOCF). Responder rates (reduction in MADRS 50\%) were significantly higher for the LUR (57\%) than for the PBO group $(42 \%$, Logistic Regression $\mathrm{p}<0.01$ ). Discontinuation rates due to adverse events were $6 \%$ for LUR and $8 \%$ for PBO. Most frequently reported adverse events were nausea (17.5\% vs. $11.0 \%)$, headache (10.4\% vs. $12.3 \%)$, and somnolence $(8.7 \%$ vs. $4.3 \%$ ) for LUR vs. PBO, respectively. 
Conclusions: In this study, adjunctive use of LUR compared to placebo significantly reduced depressive symptoms in patients with bipolar I depression who had inadequate response to either Li or VPA alone. LUR treatment also significantly improved measures of social and occupational function as well as quality of life. The tolerability and safety profile of LUR observed in this study was consistent with that reported in previous studies in schizophrenia.

Educational Objectives: After reviewing this poster, the participant may be better able to understand the potential effect of lurasidone as an adjunct to either lithium or valproate in patients with bipolar I depression.

The following information concerns a use of lurasidone that has not been approved by the U.S. Food and Drug Administration.

Trial registration: clinicaltrials.gov identifier: NCT00868452

Sponsored by Sunovion Pharmaceuticals Inc.

PS-121

Self report neuropsychiatric checklist guides rational psychopharmacology to stabilize 121 acutely mentally ill individuals in an outpatient mental health center

David R. Torres, MD; Lisa Chavez

\begin{abstract}
Self report neuropsychiatric checklist guides rational psychopharmacology to stabilize 121 acutely mentally ill individuals in an outpatient mental health center.

In Colorado, there is a movement to replace civil state hospital beds allocated for mental health center use with individuals undergoing forensic competency evaluations. This movement has led to a lack of available psychiatric beds at the State Hospital level and has increased the need for rapid stabilization of mentally individuals in emergency rooms and in mental health center settings. Innovative evidenced based treatment algorithms driven by coorborated self report scales are necessary for the optimal treatment of severe neuropsychiatric illness. Rapid pharmacologic assessment and treatment is part science and part art. Innovations and enhancements of already established treatment protocols are necessary to provide optimal health care for those in need in the community. A universal neuropsychiatric scale designed in 2009 called the Colorado Mood Rating Scale was used to assess and guide medication management of mentally ill individuals seen in a mental health center system in frontier areas of Southeastern Colorado has shown positive results in its first 5 months of implementation.
\end{abstract}

The Colorado cycling mood rating combines select questions from the Young mania rating scale, Hamilton depression scale, adult ADHD self report scale and questions related to mood stabilizer toxicity. Each of these validated scales individually has their own strengths and weaknesses when assessing human behavior and thought. A global assessment scale of thought and behavior and functioning is important to document before psychopharmacologic recommendations can be made. Speed of data accumulation allows for more time for problem solving about potential treatment recommendations. The self rating scale is 60 questions takes about 3 minutes to complete and needs corroboration from a third party. The goal of treatment was to have all individuals enter remission rapidly and treat comorbid illness including vasomotor symptoms, pain, potential seizure activity, and substance abuse concurrently. Treatment was initiated without the use of benzodiazepines, anticholinergics or opiates to preserve cognitive functioning.

The use of this universal neuropsychiatric screen to assess symptoms and guide management resulted in improvement from baseline of 10 to 50 percent. All individuals were treated to remission and 2 were hospitalized out of the group. One previously hospitalized individual has since been stabilized on lithium the other stabilized when she became compliant taking her previously prescribed medications. A functional improvement was noted in all individuals treated in the standard of care. Most of the improvement seen was in individuals with depressive symptoms. Those individuals who were treated with stimulants and wake promoters showed benefit in the realms of attention, concentration and focus.

The scale provides rapid guidance regarding percentile dose reduction of mood stabilizers when side effects are present. The scale may have clinical utility when used in consultation with primary care offices and emergency rooms when recommendations regarding medication management is required. The scale may be tested in the future with PET, FMRI and diffusion tensor imaging to establish correlation with known circuit systems which modulate human behavior and thought.

The scale guides medication management when one time test dosing of atypical antipsychotics and stimulants are used. The scale provides guidance regarding percentile dose reduction of mood stabilizers when side effects are present.

No funding 
PS-122

Objective Assessment of Sleep Quality in Adults: A Case Study

Kelly L. Olson, PhD; Scott Theirl, DC, DACNB, FACFN; David N. Baker, BSc

\begin{abstract}
:
Study Objective: In the past, there was no simple, cost effective, objective measure of sleep quality available to the clinician. The present case study begins a series of cases investigating the utility \& objectivity of measuring sleep quality via cardio-pulmonary coupling. We aimed to identify patients with sleep issues and to determine whether this objective screening technology could effectively monitor therapeutic success over time.

Methods: For this case study we assessed a male patient, 50 years old. He was examined and treated at a functional restoration clinic in Milwaukee, Wisconsin. The patient, identified as having issues with sleep, was given a thorough history and physical examination, asked to provide specimens for biochemical testing and to wear a small device at home that recorded sleep quality the same night as specimen collection. Upon receipt of the patient's laboratory workups, he was given a tailored treatment regimen aimed primarily at addressing sleep issues. The patient was treated for 13 weeks before a series of retests were conducted. Sleep and biomarker test results led to adjusted therapies and an additional 8 weeks of treatment. At week 21 another series of retests were performed.
\end{abstract}

Results: After 13 weeks of treatment the patient was retested specifically for the presence and/or improvement of sleep issues. The patient subjectively reported that he seemed to "sleep better". The objective measure of sleep quality did not strongly show evidence of this change as high frequency coupling (good, stable sleep) increased only slightly (5\%), while low frequency coupling (poor, unstable sleep) also increased (15.6\%). Together these parameters indicated overall independent sleep quality remained poor. After additional therapies were added for 8 weeks, the patient continued to report subjective improvement in his sleep. The objective sleep quality retest at week 21 supported the subjective report revealing high frequency coupling had increased (17\% from retest) and low frequency coupling decreased $(17.7 \%$ from retest).

Conclusions: Based on the case study results along with previously published data, present objective measurements were successful at identifying the presence of sleep issues. Furthermore, these independent measures provide an unbiased report of patient physiology while monitoring clinical therapeutic success.

Educational Objectives: There has been a chasm that existed between psychiatry and sleep. In-home Sleep quality screening closes this gap and arms the psychiatrist with an unbiased, more focused understanding of the characteristics involved in impaired sleep. Simple, objective measurements of sleep quality allow the clinician to more completely analyze sleep issues present in a wide range of patient populations.

Funding: This study was funded by SleepImage.

PS-123

A Multi-analyte Biomarker Approach for the Detection of Depression in a Clinical Population with Comorbid Pain John A Bilello, PhD; Bo Pi, PhD; Linda M. Thurmond, PhD; Katie M. Smith, PhD

\begin{abstract}
:
Study Objective: To determine whether a blood-based biomarker panel for depression could identify depression comorbid with chronic pain. Comorbid depression can complicate the presentation, clinical course, and response to treatment of patients with chronic pain.

Background: Patients with common mental disorders, such as depression and anxiety, have a tendency to present first to primary care exhibiting symptoms of pain and other physical complaints that appear idiopathic. Emerging evidence suggests that pathophysiologic mechanisms underlie the coexistence of depression and chronic pain. A biomarker panel and algorithm for depression diagnosis was developed consisting of nine biomarkers associated with the neurotrophic, metabolic, inflammatory or HPA axis pathways.
\end{abstract}

Methods: Patients from community psychiatric practices were enrolled per the managing clinician's discretion. The majority of patients had recurring depressive symptoms and/or were patients who were difficult to manage or non-compliant. The study enrolled a total of 98 patients, 80 of which ( $81 \%$ compliance) had a blood sample drawn. The mean age of the study population was $48.5 \pm 12.6$ with a range from $21-80$ years. Fifty-six $(70 \%)$ were female and $24(30 \%)$ were male. Normal subjects were from a cohort established previously [Papakostas, et. al. Molecular Psychiatry, 2012]. Patient samples were tested and serum levels of the nine markers measured by quantitative immunoassay; an MDD Score was determined using a proprietary algorithm.

Results: The biomarker panel showed discrimination of patients with depression $(n=80)$ from normal subjects $(\mathrm{n}=43)$ with a $\mathrm{p}$ value $=<0.00001$ and a clinical sensitivity of $90 \%$ and specificity of $87 \%$. While 16 of 80 subjects 
had no comorbidity listed, the majority of the enrolled subjects had a variety of comorbidities, with some of the most common being: arthritis, diabetes, hypertension, obesity and chronic pain. Eighteen of 80 patients had chronic pain comorbid with depression. Six of 18 had chronic pain as the only comorbidity. The remaining 12 had pain and arthritis (8); pain \& obesity (3) pain and hypothyroid (1). The MDD scores for depressed subjects with pain and at least one other comorbidity were consistent with a high probability of MDD. Nonetheless, there were differences in individual biomarker and pathway expression patterns.

Of the six patients with chronic pain only four had a likelihood of depression of $>90 \%$ and two had 10 and $33 \%$ likelihood, respectively. Conclusions: While comorbid depression can complicate the presentation, clinical course, and response to treatment of patients with chronic pain: a blood-based test is able to identify depression in patients with comorbid chronic pain.

Educational Objectives: Primary care physicians and psychiatrists have few truly objective tools to aid them in diagnosis of depression. After assimilating this material, the participants would be made aware of new tools now available to aid in depression diagnosis in chronic pain patients.

Funding: This work was funded by Ridge Diagnostics Inc. JB, BP, LT and KS are employees of Ridge Diagnostics, Inc.

\title{
PS-124
}

Gabapentin: "Off-label" for pain, anxiety and insomnia

Daniel A. Deutschman, MD, DLF, APA

\begin{abstract}
:
Objective: To assess Gabapentin's ability to replace narcotics, benzodiazepines and sedatives for pain, anxiety and insomnia in unselected adult and geriatric patients.

Method: Every adult and geriatric patient presenting with pain, anxiety or insomnia (to a psychiatry and addiction practice for a 36 month period) was placed on escalating doses of Gabapentin. No upper limit was set for individual dose or dose per 24 hours. If patients began to respond to Gabapentin, narcotics, benzodiazepines and sedatives were titrated downward and discontinued. Patients with preexisting renal disease were begun on small doses (50 to $100 \mathrm{mg}$ ). All patients were warned that they might experience sedation and/or unsteadiness, initially, as Gabapentin titrations were begun. Where this occurred, doses were reduced to improve tolerability. If therapeutic effects of Gabapentin diminished over time, doses were increased until full efficacy returned.
\end{abstract}

Results: In all, 159 consecutive adult and geriatric psychiatry and addiction patients ages 19 to 93 years were started on Gabapentin. Fewer than 5\% discontinued because of initial side effects. Some patients required very large doses (two to five times the FDA suggested maximum). Tachyphylaxis developed in some patients requiring an increase in the dose of Gabapentin to maintain symptom relief. Side effects were minimal once initial doses were tolerated. There were no safety issues.

Discussion: Absorption and Distribution: Gabapentin is reported to be subject to two transporters. One controls absorption from the gut; the other controls access to the brain (the Blood Brain Barrier). The literature suggests the maximum capacity of these transporters is $2,400 \mathrm{mg}$ to $3,600 \mathrm{mg}$ per day. Our data is at variance with these assertions.

Dose per day: Many of our patients required 6,000 to $10,000 \mathrm{mg}$ per day for sustained relief enabling them to discontinue narcotics, benzodiazepines and sedatives.

Age: Even older geriatric patients (age 80 years plus) responded well to Gabapentin, some required 6,000 mg/d or more.

Addiction: No dependency issues have surfaced with Gabapentin in our patients. Even patients who had been addicted to narcotics, benzodiazepines and sedative for decades were successfully titrated off of these agents using Gabapentin.

Tolerability: Once any initial side effects had passed, tolerability was excellent. Cognitive effects were unusual. Edema was not seen.

Safety: The literature reported recently that "accidental death and suicide from narcotics, benzodiazepines and sedatives has now surpassed highway fatalities". Gabapentin has no suicide potential. Suicide attempts with as much as $91,000 \mathrm{mg}$ were of no lasting consequence (patient slept for 36 hours).

Comment: This was an open label, uncontrolled, voluntary, "off-label", observational trial. It was meant as an initial test of concept. More rigorous studies should be conducted to see if the results presented here can be duplicated. 
Conclusion: Gabapentin is able to replace narcotics, benzodiazepines and sedatives for pain, anxiety and insomnia in unselected adult and geriatric psychiatry and addiction patients. Tolerability and efficacy are high.

Educational Objective: After reviewing this material, the participant should understand the expanded value of Gabapentin for pain, anxiety and insomnia.

Funding: no funding.

References:

1. Fernandez MC, Walter FG, et al (1996) Gabapentin, . intoxication: elevated blood levels with mild clinical effects. J Toxicol Clin Toxicol 34:437-439. (Overdose with 91 grams of Gabapentin).

2. Angelotti CP, Fauman E (2007) Pharmacology and mechanism of action. Epilepsy Res 2007, 73(2): 137-150 (Calcium Channel Subunits and binding site for gabapentin).

3. Morrell MJ, McLean MJ, et al The Steps Study Group, Seizure 2000, 9(4): 241-248 (Efficacy of gabapentin in a large multicenter study) 4. Hendrich J, VanMinh AT, et al Pharmacological disruption of calcium channel.gabapentin. Proc Natl Acad Sci USA 2008, 105(9): 3628-3633. (Mechanism of action of Gabapentin on Calcium Channels) 5. Anton RF, et al Gabapentin for Alcohol Withdrawal, Am J Psychiatry. 2011;168:709-717 6. Striano P, Striano S, Gabapentin and Benzodiazepine Withdrawal, Drugs Today (Barc), 2008 May;44(5):353-368

PS-125

Selegiline Transdermal System (STS) in Patients with Recurrent, Unipolar Major Depression: A Post-hoc Analysis of Two Randomized, Double-blind Studies

Donald Robinson, MD; Kimberly Blanchard Portland, PhD

\begin{abstract}
:
Study Objective: Major depressive disorder (MDD) is a debilitating, chronic condition that can recur in $>75 \%$ of patients and last 2 years or longer in $15 \%$ to $20 \%$ of patients. Selegiline transdermal system (STS) is a well-tolerated acute and maintenance treatment for MDD. STS delivers sustained blood levels of monoamine oxidase inhibitor (MAOI) directly into the systemic circulation, avoiding the need for a tyramine-restricted diet at the $6 \mathrm{mg} / \mathrm{day}$ dose. The objective of this post-hoc analysis was to examine the efficacy of STS vs placebo in recurrent, unipolar major depression.
\end{abstract}

Method: Data from two pivotal (one 6 week and one 8 week), randomized, double-blind, placebo-controlled clinical trials of STS were pooled for this analysis $(\mathrm{N}=433)$. Single vs recurrent depressive episodes were coded by the investigator at the baseline assessment. An analysis of covariance (ANCOVA) model, controlling for baseline severity and study, was used to evaluate the effect of treatment as measured by the 28-item Hamilton Rating Scales for Depression (HAMD-28) and the Montgomery-Åsberg Depression Rating Scale (MADRS) at study endpoint. Chi-square analysis was used to assess differences between STS and placebo in rates of remission.

Results: Nearly three quarters of the patients $(70.4 \% ; n=305)$ had "recurrent depression" (mean [SD] baseline HAMD-17 score was $23.1[ \pm 2.4]$ ). In patients with recurrent depressive episodes, STS showed significantly greater improvement at endpoint on MADRS and HAMD-28 total scores vs patients receiving placebo $(\mathrm{p}<0.001)$. Additionally, remission rates as defined by endpoint HAMD-28 and MADRS scores were significantly greater in patients with recurrent depression treated with STS than placebo. HAMD-28 remission rates were $21.8 \%$ vs $10.1 \%$ (STS vs placebo; $\mathrm{p}<0.01$ ) and MADRS remission rates were $27.9 \%$ vs $13.3 \%$ (STS vs placebo; $\mathrm{p}<0.01$ ).

Conclusion: STS is an effective treatment for patients with recurrent episodes of unipolar, major depression. Interestingly, treatment effects were not as strong in patients with single episode depression.

Educational Objective: After studying this material, participants should be better able to understand the efficacy of a transdermal MAOI for patients with recurrent, unipolar major depression.

Funding: This study was supported by funding from Mylan Specialty L.P.

PS-126

Selegiline Transdermal System (STS) for Major Depressive Disorder (MDD): Use Pattern, Adherence, and Effect on Health Service Expenditures

Lawrence J. Cohen, PharmD; David A. Sclar, BPharm, PhD; Kimberly Blanchard Portland, PhD

Abstract:

Study Objectives: There is renewed interest in the use of MAOIs for the treatment of MDD, specifically MDD with atypical features, anxious features, and treatment-resistant depression (TRD). The American Psychiatric 
Association and the British Association for Psychopharmacology guidelines for depression list use of MAOIs as an option for TRD. Selegiline is an irreversible inhibitor of MAO enzymes. The selegiline transdermal system (STS) bypasses first-pass metabolism, and thereby inhibits MAO in the brain without significantly inhibiting MAO in the gut. The present study was designed to discern: (i) the pattern (sequence) of use of STS in MDD; (ii) level of adherence to STS as compared to alternative antidepressant pharmacotherapy regimens; and (iii) the effect on health service expenditures.

Method: This research employed patient-level data abstracted from domestic (U.S.) longitudinal archives (Medicaid; Medicare; managed care), cross-sectional surveys (U.S. National Center for Health Statistics), and the published literature. MDD was defined as ICD-9-CM codes 296.2, 296.3, 300.4 or 311. Treatment failure (TF) was defined as receipt of $<90$ days of initial antidepressant pharmacotherapy. For longitudinal analyses primary criterion were: (1) ambulatory patients aged 18 through 75 years; (2) continuous enrollment of 18 months (six months prior to an ICD-9-CM code for MDD (index date); 12 months post index date); (3) no ICD-9-CM code(s) for co-morbid mental illness; (4) initial antidepressant pharmacotherapy: SSRI, SNRI, or STS. Multivariate logistic regression and log-transformed multivariate linear regression were used to assess sequential use of antidepressant pharmacotherapy, level of adherence, and health service expenditures (intent-to-treat [ITT] and propensity-score basis), respectively.

Results: For the majority of patients, STS was prescribed as a second or third treatment option for MDD. Specifically, post TF with one SSRI or SNRI (22\%); post TF with a second SSRI or SNRI (67\%); or post TF with an SSRI or SNRI and augmentation with an atypical antipsychotic (11\%). STS was associated with a greater probability of receipt of 60,90 , or 180 days of pharmacotherapy $(p<0.05)$. Use of STS resulted in reduced $(p<0.05)$ or comparable $(\mathrm{p}=\mathrm{NS})$ health services expenditures dependent on the use pattern sequence post the index date.

Conclusions: Treatment failure is associated with increased health service expenditures. Use of STS post TF (ITT or propensity-score basis) resulted in increased adherence and comparable or reduced health service expenditures. After an antidepressant TF, early use of STS may be warranted.

Educational Objective: After studying this material, participants should be better able to understand patterns of use and in healthcare expenditures with a transdermal MAOI in patients with MDD.

Funding: This study was supported by funding from Mylan Specialty L.P.

\title{
PS-127
}

Predictors of Relapse in a Fixed-dose, Randomized, Double-blind, 52-week Relapse Prevention Trial of Selegiline Transdermal System (STS) in MDD

Saeheon Jang, MD; Sungwon Jung, MD, PhD; Chi-Un Pae, MD, PhD; Youngmyo Jae, MD, PhD;

Kimberly Blanchard Portland, PhD; Ashwin A Patkar, MD, MRC, Psych

\begin{abstract}
:
Study Objective: The variability in treatment relapse in major depressive disorder (MDD) has led to investigations of the relevance of patient characteristics. We investigated clinical characteristics predictive of relapse in a 52-week controlled trial of selegiline transdermal system (STS).

Method: After 10 weeks of open-label stabilization with STS, 322 remitted patients with MDD were randomized to 52-weeks of double-blind treatment with STS $(6 \mathrm{mg} / 24 \mathrm{hr})$ or placebo. Relapse was defined as Hamilton Depression Rating Scale (HAMD-17) score of ?14 and a CGI-S score of ?3 with at least 2-point increase from the beginning of double-blind phase on 2 consecutive visits. Pretreatment demographics, illness course, treatment resistance and symptom domains were studied to identify predictors of relapse.
\end{abstract}

Results: Significantly fewer STS patients $(16.8 \%)$ relapsed compared to placebo $(30.7 \%)(\mathrm{p}<0.005)$ and had a significantly longer time to relapse than did placebo $(\mathrm{p}<0.005)$. Baseline total HAMD-28 score, somatic anxiety, recurrent MDD, atypical depression, and analgesic use (celecoxib, naproxen) predicted relapse. Significant predictors of differential outcome were identified: 1) high baseline HAMD-28 score $(\mathrm{p}<0.001)$, high somatic anxiety $(p<0.05)$ and celecoxib use $(p<0.05)$ predicted relapse with STS; 2$)$ atypical symptoms $(p<0.05)$, recurrent episodes $(\mathrm{p}<0.05)$ and naproxen use $(\mathrm{p}<0.01)$ predicted relapse with placebo.

Conclusions: For patients on STS, higher baseline depression severity, somatic anxiety, or receiving celecoxib predicted relapse, while predictors of relapse with placebo were atypical or recurrent depression or naproxen use. The results provide indirect evidence of treatment specificity by identifying characteristics which may be of value in selection of patients for STS treatment. 
Educational Objective: After studying this material, participants should be better able to understand predictors of relapse with a transdermal MAOI in patients with MDD.

Funding: This study was supported by funding from Mylan Specialty L.P.

\title{
PS-128
}

Efficacy and Safety of Selegiline Transdermal System for Atypical Depression: Pooled Analysis of Short-Term, Placebo-Controlled Trials

Ashwin A Patkar, MD, MRC, Psych; Saeheon Jang, MD; Kimberly Blanchard Portland, PhD; Terry Painter; Sungwon Jung, MD, PhD; Chi-Un Pae, MD, PhD; J. Craig Nelson, MD

\begin{abstract}
:
Study Objective: Monoamine oxidase inhibitors (MAOIs) have been reported to be of particular benefit in major depressive disorder (MDD) with atypical features. Selegiline transdermal system (STS) is an MAOI that bypasses the gastrointestinal system and reduces the risk for dietary interactions; particularly at the lowest dose. We investigated the efficacy and safety of STS in MDD with atypical features.

Method: We conducted a post-hoc, pooled data analysis of four short-term, randomized, double-blind, placebocontrolled trials with STS (three 8-week studies: two fixed-dose trials with 3 or $6 \mathrm{mg} / 24 \mathrm{hr}$ and one flexible dose trial with 6, 9 and $12 \mathrm{mg} / 24 \mathrm{hr}$, and one 6-week trial: fixed dose $6 \mathrm{mg} / 24 \mathrm{hr}$ ). In accordance with published literature, atypical subtype was defined as presence of at least one item with a score of 2 or greater from items 22-26 on the 28-item Hamilton Depression Rating Scale (HAMD-28), and a maximum score of one point for items 6 (insomnia late), 12 (somatic symptoms, gastrointestinal), and 16 (loss of weight) to exclude vegetative features of melancholic depression. Response was defined as ?50\% reduction in HAMD-28 scores and remission as ?10 HAMD-28 score at the end of the trials. We used repeated measures ANCOVA and Chi-square tests to compare between-group differences on the primary outcome measure of mean change in total HAMD-28 score from beginning to end of treatment. Descriptive statistics were used to examine rates of adverse events in the two groups.
\end{abstract}

Results: STS ( $n=667)$ significantly decreased HAMD-28 total score compared with placebo $(n=516)$ from beginning to end of treatment $(-11.2 \pm 9.6$ vs $-9.9 \pm 9.6, \mathrm{~F}=5.846, \mathrm{p}=0.016)$. Two hundred and eighty six $(24.2 \%)$ subjects met the definition of atypical subtype. STS showed comparable efficacy between the atypical $(n=158)$ and non-atypical $(n=509)$ subtypes with respect to total HAMD-28 score change (12.2 \pm 9.6 vs $10.9 \pm 9.6, \mathrm{~F}=0.135, \mathrm{p}=0.714)$, response rates (40.3\% vs $41.9 \%, \mathrm{p}=0.729)$ and remission rates $(27.3 \%$ vs $28.4 \%, \mathrm{p}=0.809)$ respectively. Discontinuation rates were similar in the atypical $(12.7 \%)$ and non-atypical $(9.2 \%)$ subtypes $(\mathrm{p}=0.211)$. The atypical and non-atypical groups were comparable in rates of skin reactions $(25.9 \%$ vs $28.5 \%)$, insomnia $(7.6 \%$ vs $10.6 \%)$ and headache $(6.3 \%$ vs $5.7 \%)$. We reanalyzed the data excluding subjects given the $3 \mathrm{mg} / 24 \mathrm{hr}$ dose $(\mathrm{n}=150,10.8 \%)$, because this dose was determined not to be effective for MDD and thus was not submitted to the FDA. The findings remained similar to the original pooled analysis.

Conclusions: In this pooled analysis, STS appears to be efficacious for patients with atypical depression, a historically difficult-to-treat subtype of MDD. STS was equally efficacious in atypical and non-atypical subtypes of MDD. These findings are consistent with results of a post-hoc analysis of the open-label phase of a relapse prevention trial of STS. Prospective trials are necessary to confirm these findings.

Educational Objective: After studying this material, participants should be better able to understand the efficacy of a transdermal MAOI in patients with atypical depression.

Funding: This study was supported by funding from Mylan Specialty L.P.

\section{PS-129}

Predictors of Response and Remission During an Open-label 10-week Trial with Selegiline Transdermal System (STS) in Major Depressive Disorder

Sungwon Jung, MD, PhD; Saeheon Jang, MD; Chi-Un Pae, MD, PhD; Prakash S. Masand, MD; Rob Mariani; Kimberly Blanchard Portland, PhD; Ashwin A Patkar, MD

\footnotetext{
Abstract:

Study Objective: Patient and treatment characteristics that influence treatment response and remission in major depressive disorder (MDD) are of clinical interest. This post-hoc analysis investigated clinical characteristics predictive of response and remission in a 10-week, open-label trial of selegiline transdermal system (STS).
}

Method: The data analyzed included 10 weeks of open-label treatment with $6 \mathrm{mg} / 24 \mathrm{hr}$ of STS in patients with MDD. This was the stabilization phase of a 52-week, placebo-controlled, double-blind relapse prevention trial with STS. 
Response was defined as $250 \%$ reduction in Hamilton Depression Rating Scale (HAMD-17) score and remission was defined as HAMD-17 score of ?10. Pretreatment demographics, illness course, treatment resistance and symptom domains were studied to identify predictors of response.

Results: 675 patients entered the trial and 366 completed open-label treatment. Dropout rate was 54\%. Response rate was $53.3 \%$ and remission rate was $47.1 \%$ by the end of 10 weeks. Early response (within first 2 weeks of treatment) $(p<0.005)$, retardation $(p<0.05)$, sexual difficulties $(p<0.005)$ and hypnotic use $(p<0.05)$ were significant predictors of response. The same factors also significantly predicted remission. Subjects with atypical, melancholic or, anxious features had comparable response and remission rates on STS to those without those features.

Conclusions: Early response was a strong predictor of end of treatment response and remission with STS. Retardation and sexual difficulties also predicted outcome with STS. Patients with atypical or melancholic features responded equally well. The results demonstrate patient characteristics that may be helpful for clinicians while treating patients with STS.

Educational Objective: After studying this material, participants should be better able to understand predictors of response and remission with a transdermal MAOI in patients with MDD.

Funding: This study was supported by funding from Mylan Specialty L.P.

\title{
PS-130
}

Selegiline Transdermal System (STS) for MDD with Atypical Features: A Post-hoc Analysis of Data from an Open-label, 10-week Trial

Saeheon Jang, MD; Sungwon Jung, MD, PhD; Chi-Un Pae, MD, PhD; Kimberly Blanchard Portland, PhD;

Rob Mariani; Terry Painter; Ashwin A Patkar, MD

\begin{abstract}
:
Study Objective: Approximately 36\% of patients with major depressive disorder (MDD) may have atypical features, which may be associated with poorer long-term prognosis. Monoamine oxidase inhibitors (MAOIs) have been shown to be efficacious in treating MDD with atypical features. Selegiline transdermal system (STS) is an MAOI that bypasses the gastrointestinal system, thereby reducing the risk of dietary interactions. We investigated the efficacy of STS in MDD patients with atypical features.

Method: Data were analyzed from a 10-week, open-label treatment phase of STS $6 \mathrm{mg} / 24 \mathrm{hr}$ in patients with MDD. This was the stabilization phase of a 52-week, placebo-controlled, double-blind relapse prevention trial with STS. Atypical subtype was defined as at least one score of 2 for items 22-26 on the 28-item Hamilton Depression Rating Scale (HAMD-28). Response was defined as ?50\% reduction in the HAMD-17 score and remission as a HAMD-17 score of $<10$ at 10 weeks. We used repeated measures ANOVA and t-tests to compare between-group differences.

Results: 675 patients entered the open-label phase trial. $371(55 \%)$ of patients met criteria for having "atypical features". In the total sample there was a significant reduction in HAMD-28 scores from baseline to end of treatment (mean change $=13.7, C I=13.0$ to $14.3, p<0.001$ ). There were no significant differences in mean HAMD-28 change from baseline to end of treatment in atypical (mean change $=-13.80, \mathrm{SD}=7.3$ ) vs nonatypical subgroups (mean change $=13.54, \mathrm{SD}=7.6)(\mathrm{p}=0.69)$. Response and remission rates were $62.5 \%$ and $46.5 \%$, respectively, in the atypical group and $61.8 \%$ and $48 \%$, respectively, in the nonatypical group.
\end{abstract}

Conclusion: In this post-hoc analysis, STS appeared to be equally efficacious in atypical and nonatypical subtypes of MDD. Further analysis of data from controlled trials is needed to confirm the findings.

Educational Objective: After studying this material, participants should be better able to understand the efficacy of a transdermal MAOI for patients with atypical depression.

Funding: This study was supported by funding from Mylan Specialty L.P.

\section{PS-131}

A trans-diagnostic observation of the efficacy of the Trial-Based Thought Record in changing negative core beliefs and reducing self-criticism

Irismar Reis de Oliveira, MD; Érica P. Duran; Michella Velasquez

Study objectives: Trial-based therapy (TBT) is a new psychotherapeutic approach whose foundation is in cognitive therapy (CT). A major goal of CT is to restructure negative core beliefs (CBs), considered the best prevention 
against relapses in several psychiatric diagnoses. The efficacy of the trial-based thought record (TBTR) - the main technique used in TBT -, in decreasing the credit given by patients to negative CBs and corresponding emotions, was evaluated in this study. In view of the fact that the published preliminary data, involving 166 patients, did not show any difference in outcomes between patients treated with TBTR, using the empty chair format relative to the conventional format, nor was any difference found regarding therapists exposure (short- vs. long-term) to the approach, we decided to investigate this further with a larger sample.

Method: Adult patients $(n=259)$, having any diagnosis, were submitted to a one-session simulation of a legal trial and their adherence to negative CBs and corresponding emotions after each step of the TBTR technique first use. TBTR comprises 7 steps: the identification of the CB (investigation), prosecutor's plea, defense attorney's plea, prosecutor's second plea, defense attorney's second plea, jury's verdict, and preparation for the appeal. Comparisons involved TBTR used in the empty chair format vs. conventional format, short-term vs. long-term exposure of therapists and completers vs. non-completers. A LOCF approach was used for incomplete data.

Results: Significant reductions in percent values both in the credit given to the CBs and in the intensity of the emotions were observed after the first and second defense attorney pleas, as well as after jury's verdict and initial preparation for the appeal $(\mathrm{p}<.001)$, relative to baseline (investigation phase, 1st step). Significant differences also emerged between the defense attorney's first and second pleas and between the defense attorney's second plea and jury's verdict, as well as preparation for the appeal (all $\mathrm{p}<.001$ ). Similarly, a significant difference between percentages presented by patients submitted to TBTR used in the empty chair format relative to the conventional format was found after verdict $(\mathrm{p}=.02)$, preparation for the appeal $(\mathrm{p}=.05)$, and overall improvement $(\mathrm{p}=.03)$, regarding intensity of the emotion. Also, significant differences were found between these groups in regard to exposure of the therapist to the approach $(\mathrm{p}<.05)$, and completion of the technique's use during the session $(\mathrm{p}<.001)$.

Conclusions: TBTR may help patients reduce attachment to negative CBs and corresponding emotions, confirming our preliminary observations. Outcomes were significantly favorable regardless of the format use and therapists' level of exposure to TBTR. However, contrary to our previous findings, significant differences emerged in both comparisons, indicating that the empty chair format is more efficacious than the conventional format in reducing the intensity of corresponding emotions. Outcomes observed in patients treated by long-term exposed therapists was significantly better in this regard.

Educational objectives: After reading this poster, the participant should be able to recognize the importance of CBs in psychiatric disorders and the importance of restructuring such cognitions.

Funding: This research was not funded. 